\title{
An Improved Design of Low-Cost Wind Tunnel for Educational Purpose in Fluid Dynamics
}

\begin{abstract}
Sharul Sham Dol
Department of Mechanical Engineering, Abu Dhabi University, P.O. Box 59911, Abu Dhabi, UAE

Abstract: The development of wind tunnel has great favor in contributing towards educational and research purposes in fluid dynamics. The commercial wind tunnel is expensive and only limited to highly-focus researchers and exclusive institutions. This limitation could hinder the progress in fluid dynamics interest among students. The project aims to design, develop, and construct a cost-effective open-circuit wind tunnel, which is able to conduct small-scale experiments in aerodynamics related problems. The previously constructed wind tunnel was improved by utilizing the settling chamber, curved-wall contraction cone and circular diffuser to enhance airflow quality. Experimental and simulation outcomes verify that the modified design is able to achieve higher flow velocity with lower turbulence intensity in the test section. Overall, it can be concluded that the objectives of the projects are accomplished and the methodology can be applied easily by engineering instructors and students for cheaper but reliable option in designing wind tunnel.
\end{abstract}

Keywords: aerodynamics; honeycomb; turbulent intensity; wind tunnel

\section{Introduction}

Knowledge on turbulent flows is both fascinating and complex. The information on these unsteady and chaotic flows serve as fundamental understanding to fluid dynamics field, which is one of the core courses to many engineering programs such as mechanical, petroleum, chemical, civil, aeronautics and aerospace. The topic often involves dynamics of fluid flow that requires flow visualization to comprehend theoretical flows. Normally, the fluid dynamics instructors use the computational fluid dynamics (CFD) simulation for simple case application problems to visualize flow phenomena to students. There are limitations on numerical approach as it involves approximations and it lacks physical depth.

The development of wind tunnels give researchers an idea how flows behave in actual. There are various different design types of wind tunnel for different applications as no single tunnel fits for all purposes. Wind tunnels can be classified based on airflow speed in test section or based on design. An advanced commercial grade wind tunnel is very expensive and exclusively available only to research-focused universities [1-4]. Thus, this project aims to design, develop, and construct a cost-effective open-circuit wind tunnel, which is able to conduct small-scale experiments accurately in any fluid dynamics course to study aerodynamics problems for educational purpose or classroom activities. This wind tunnel mainly focuses on testing any scaled-down model (i.e. cars, airplanes, dams, bridges, towers and buildings).

For the wind tunnel test section, the flow quality is subjected to temporal and spatial aspects of the flow [5]. Deviation from spatial uniformity is undesirable as it would affect the integrity of flow consequently results in poor test outcome. Turbulence intensity of less than $2 \%$ at working speed of $6 \mathrm{~m} / \mathrm{s}$ to $8 \mathrm{~m} / \mathrm{s}$ would be considered reasonable for testing without compromising the flow unsteadiness.

\section{Methodology}

The improved model of wind tunnel design consists of a settling chamber, curved-wall aluminum contraction cone with trip wire, plexiglass test section, circular aluminum diffuser and 12-inch gable fan. A settling chamber consists of honeycomb and screens that act as a flow straightener where they allow the air to enter the tunnel in one direction parallel to fluid flow motion. Trip wire installation to contraction cone wall boundary is able to minimize the fluctuations intensity by setting the transition initiation point (Figure 1). This produced more uniform flow in the test section. The cross-sectional area of a contraction decreases towards 
upstream of the test section. This increases flow mean velocity and reduces pressure loss consequently as the settling chamber is in a low speed region. The length of contraction cone should be sufficiently long to avoid flow separation but also should be kept short enough to minimize the boundary layer growth and vortices effects apart from reducing the cost and space. The cross-sectional area of a contraction decreases towards downstream of the diffuser. This decreases the airflow velocity as it flows towards wind tunnel exit. Flow quality in the diffuser dependent upon geometry parameter such as area ratio, diffuser angle, wall contour and cross-sectional shape. Curved wall diffuser is effective in reducing the high-speed flow at outlet.
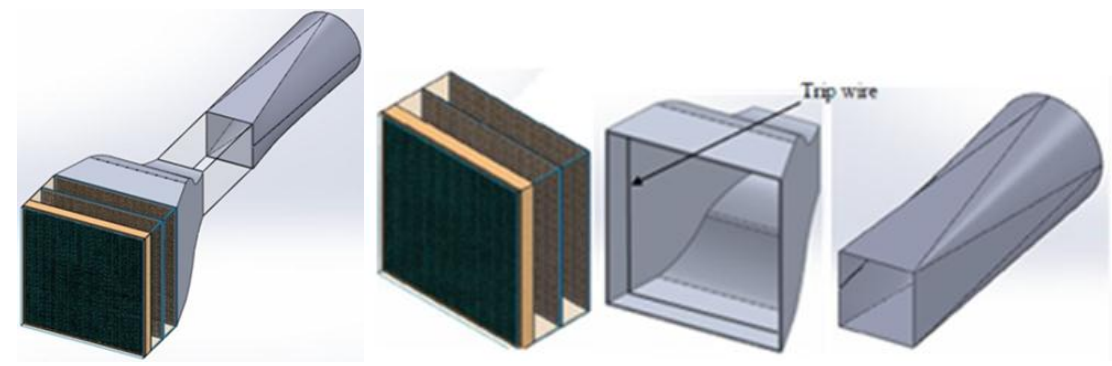

Fig. 1 Design of wind tunnel that includes settling chamber, contraction cone and diffuser

The designs of wind tunnel are verified and improved using Ansys Fluent 14.5 CFD simulation. The results are validated by the anemometer probe measurements for both instantaneous velocity and time-averaged velocity for turbulence statistical data. Over a specific period of time, the velocity, time averaged velocity and root mean square velocity at a given point were determined by using equations by using equation 1 to 4 respectively.

$$
\begin{aligned}
& U=U_{\text {mean }}+U_{\text {rms }} \\
& U_{\text {mean }}=\frac{1}{n} \sum_{i}^{n} U_{i} \\
& U_{\text {rms }}=\sqrt{\frac{1}{(n-1)} \sum_{i=1}^{n}\left(U_{i}-U_{\text {mean }}\right)^{2}} \\
& T u=\frac{U_{\text {rms }}}{U_{\text {mann }}}
\end{aligned}
$$

Where $U$ is the velocity $(\mathrm{m} / \mathrm{s}), U_{\text {mean }}$ is the time averaged velocity $(\mathrm{m} / \mathrm{s}), U_{\text {rms }}$ is the root mean square or standard deviation of air speed $(\mathrm{m} / \mathrm{s})$, and $n$ is the number of samples.

\section{Result and Discussion}

Fig .2 and Fig. 3 show the simulation results; velocity magnitude and turbulence intensity in contour form for the modified wind tunnel model.

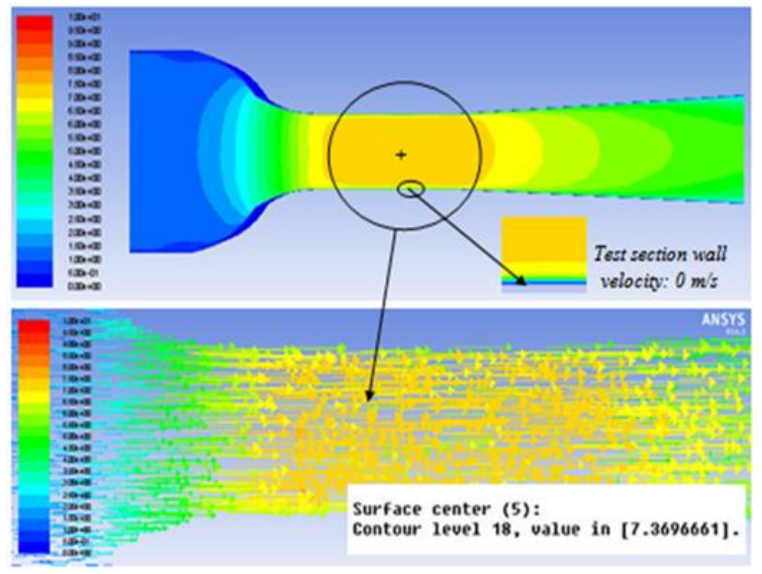

Fig. 2 Contour of velocity magnitude and velocity vector (below) 


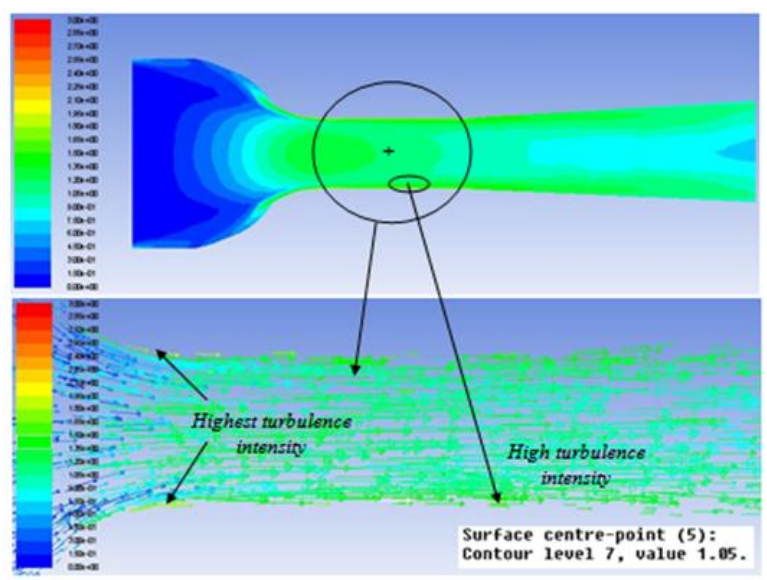

Fig. 3 Contour of turbulence intensity and turbulence vector (below)

Velocity contour shows bulk profile of air flow velocity where it increases along the contraction cone and retains as it enters the test section before it gradually decreases as it enters the diffuser. The middle section of test section has more uniform velocity magnitude as it is free from boundary layer, wake and vortices. As air flows near to contraction cone inlet, velocity increases more rapidly which results in higher velocity magnitude in the test section. Diffuser also shows high efficiency in reducing air flow velocity in a shorter distance.

Figure of velocity vector shows air particle displacement and magnitude at each point. It appears that air flows uniformly in the test section. At the interface region where air flows through contraction outlet and test section inlet, the arrows show uniform air flow pattern. This indicates that the flow does not experience sudden increase in velocity and does not generate vortices along the path. Velocity magnitude ranges between $6.50 \mathrm{~m} / \mathrm{s}$ to $7.50 \mathrm{~m} / \mathrm{s}$. This is higher than existing wind tunnel where the velocity magnitude ranges between $6.28 \mathrm{~m} / \mathrm{s}$ to $6.65 \mathrm{~m} / \mathrm{s}$ [8]. For second model, velocity magnitude at the center of the test section the is $7.37 \mathrm{~m} / \mathrm{s}$, signifies by contour level 18.

Turbulence contour shows bulk profile of turbulence intensity in \% where it increases uniformly along the contraction cone and it remains increasing as it enters the test section. As air flow nearer test section center, turbulence intensity decreases and it retains before it gradually decreases as it enters the diffuser. Figure of turbulence vector shows air particle displacement and magnitude at each point. It can be observed that the turbulence intensity is maximum at the interface of contraction cone and test section. This is because the flow fluctuates vigorously due to increase in speed as a result of increase in cross-sectional area.

The turbulence intensity at different point in the test section also varies. Near to test section wall, the turbulence intensity is high due to unstable flow as a result of boundary layer thickness and skin friction. Though, the flow at the outer layer of the boundary layer appears to be less fluctuating. As air flows near to the middle of the test section where the flow is fully developed, steadier and the boundary layer appeared to be fluctuating lesser. The turbulence intensity in the test section ranges from approximately $0.90 \%$ to $1.35 \%$ for which of lower than previous wind tunnel, $2.72 \%$ to $3.39 \%$ [6]. For the second model, turbulence intensity velocity at the center of the test section the is $1.05 \%$, signifies by contour level 7 . Simulations results justify that the design is viable to be implemented for second model.

The analysis of experimental results focuses on main area of discussion of this project which is; the performance of wind tunnel before and after modifications. Graph of air flow velocity versus time for both wind tunnels (Fig. 4) shows that velocity fluctuates rapidly in variation of time. However, there is an extremely noticeable dissimilarity in its velocity fluctuation. Series of data for first model occupies the lower velocity region of the graph whilst for second model, the data occupies the higher velocity region of the graph. It can be observed that for the first model, the velocity is noticeably very inconsistent as the it fluctuates dramatically where the velocity ranges from $4.95 \mathrm{~m} / \mathrm{s}$ to $5.50 \mathrm{~m} / \mathrm{s}$ in $128 \mathrm{~s}$. This yields percentage difference of $10.53 \%$. In contrary for second model, the velocity fluctuates moderately where the velocity ranges from $5.96 \mathrm{~m} / \mathrm{s}$ to 6.08 $\mathrm{m} / \mathrm{s}$ in $128 \mathrm{~s}$. This yields percentage difference of $1.99 \%$ which is much lower compared to previous model. 
This difference in inconsistency for both models results in significantly different mean velocity and turbulence intensity.

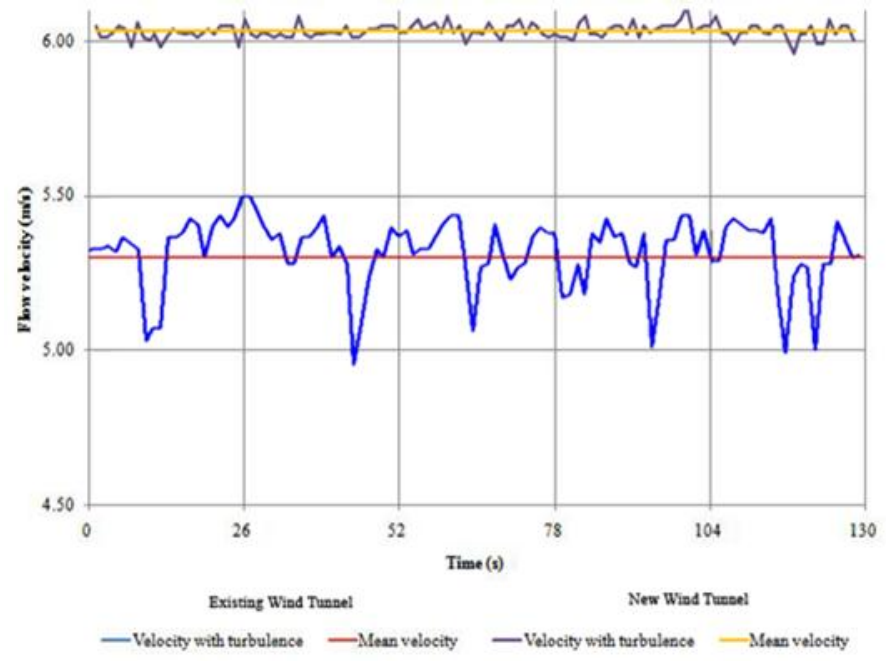

Fig. 4 Graph of velocity magnitude (m/s) against time (s) for new and existing wind tunnel

Table I shows tests results for both wind tunnels. Based on these results, velocity magnitude yield for second model is $6.05 \mathrm{~m} / \mathrm{s}$ which is $13.99 \%$ higher than velocity magnitude yield in previous wind tunnel, $5.29 \mathrm{~m} / \mathrm{s}$. The turbulence intensity for newer wind tunnel is $0.40 \%$ which is $80.30 \%$ lower than turbulence intensity for previous wind tunnel, $2.03 \%$. This signifies the newer wind tunnel is able to produce better flow quality compared to the previous wind tunnel as higher velocity with lower turbulence intensity is exceptionally desired in the test section of the wind tunnel. As the existing test section and gable fan were re-used in order to maintain test parameter such as dimension of test area and fan speed, thus best explanation of this fine results might be due to the utilization of new components for the wind tunnel.

Table I Summary Of Test Results

\begin{tabular}{|l|l|l|}
\hline Wind tunnel & $\begin{array}{l}\text { Velocity magnitude } \\
(\boldsymbol{m} / \boldsymbol{s})\end{array}$ & Turbulence Intensity (\%) \\
\hline $\begin{array}{l}\text { Existing wind tunnel } \\
\text { (Before modifications) }\end{array}$ & 5.29 & 2.03 \\
\hline $\begin{array}{l}\text { New wind tunnel } \\
\text { (After modifications) }\end{array}$ & 6.05 & 0.40 \\
\hline Percentage vary (\%) & 13.99 & 80.30 \\
\hline
\end{tabular}

Second model utilizes settling chamber, curved-wall contraction cone and circular diffuser which greatly facilitated the enhancement of air flow quality. The installation of settling chamber at contraction cone inlet which replaced one single screen of existing wind tunnel helped suppressed the cross velocity of extremely unsteady air condition. The honeycomb itself straightened the air flow, reduced turbulent length scales, lateral turbulence components and mean flow gradients and eliminated the swirling at the inlet. Subsequently, straightened air flew through screens where the velocity was uniformed. This provided the contraction cone a more uniform and steady air condition thus resulted in less exertion required by the contraction cone to reduce the turbulence in terms of percentage of free stream velocity.

Design contraction cone also played fundamental role in improving flow uniformity and reducing turbulence intensity in the test section. The first model employs contraction cone with symmetric cubic wall shape where boundary layer separation was likely to occur near the outlet of non-circular cross-section outlet. This is highly undesirable as flow separation caused fluid flow detachment form contraction wall and took the forms of eddies and vortices. This consequently interrupted flow quality in the contraction cone and increased turbulence intensity in the test section. In contrary, the second model employ curved-wall contraction cone with trip wire 
installed to contraction cone wall boundary. Apart from its ability to produce high air flow velocity, the separation was also unlikely to occur.

Trip wire installation is able to minimize the fluctuations intensity by setting the transition initiation point. The pressure distribution in contraction cone was altered where the adverse pressure gradient was moved by the trip wire toward the inlet of the contraction cone. This produced more uniform flow in the test section. The trip wire also promoted the turbulence within the boundary layer in order to yield more efficient momentum mixing. This essentially weakened the formation of vortices and avoided the incipient separations. Thus as air reached the test section inlet, the air flow was already in uniform, steady and high velocity condition.

Diffuser design also dictates the flow quality in the test section. Sharp edges diffuser of first wind tunnel was likely caused flow separation which produced vortices that interrupted the integrity of flow quality in the test section. By utilizing circular diffuser, the flow separation in the diffuser can be avoided. Apart from that, unlike symmetrical cubic diffuser, circular diffuser has better ability to minimize losses in the wind tunnel. It efficiently recovered the pressure and reduced air velocity coming from the test section consequently increased the air flow velocity in the test section. This elucidates higher mean velocity magnitude yield for second model.

In addition, smooth surface of aluminum compared to plywood might also contribute to results as it reduces friction that slows down the phase of air flow. The combination of curved-wall aluminum contraction cone and circular aluminum diffuser had proven to produce higher velocity magnitude with low turbulence intensity in the test section. Overall, evidently it can be deduced that the utilization of new component such as settling chamber, curved-wall contraction cone with trip wire and circular diffuser able to promote better flow quality. Higher flow velocity with lower turbulence intensity is extremely desired in the test section. Thus the implementation of new design is more effective as it yields better flow quality, hence capable to accomplish high quality result. A more accurate prediction of test subject elements can be achieved therefore reduces the percentage of uncertainty. This is extremely advantageous when testing a subject.

\section{Conclusion}

The development of wind tunnel has great favor in contributing towards educational and research purposes. Overall, it can be deduced that the utilization of new component such as settling chamber, curved-wall contraction cone with trip wire and circular diffuser are able to promote better flow quality. Higher flow velocity with lower turbulence intensity are achieved in the test section. Thus, the implementation of new design in the final tunnel construction is more effective as it yields better flow quality. The wind tunnel is equipped to operate and to be used for educational and research purposes. On the whole, it can be concluded that the objectives of the projects were accomplished.

\section{References}

[1] Bradshaw, Peter and Pankhurst RC 1964 The design of low-speed wind tunnels Progress in Aerospace Sciences 5 169.

[2] Moonen, Peter, Blocken, and Jan Carmeliet. 2007. Indicators for the Evaluation of Wind Tunnel Test Section Flow Quality and Application to a Numerical Closed-Circuit Wind Tunnel. Journal of Wind Engineering and Industrial Aerodynamics 95, no. 9-11: 1289-314.

[3] Kulkarni, NirajanSahoo and Sandip D. Chavan. 2010. "Simulation of honeycomb-screen combinations for turbulence management in a subsonic wind tunnel." Journal of Wind Engineering and Industrial Aerodynamics.99: 37-45. Dol:10.1016/j.jweia.2010.10.006. https://doi.org/10.1016/j.jweia.2010.10.006

[4] Arifffuzzaman, and Mohammad Mashud. 2012. "Design Construction and Performance Test of a Low Cost Subsonic Wind Tunnel." Journal of Engineering. 2(10): 83-91.

[5] Ghorbanian, Mohammad Reza Soltani and MojtabaDehganManshadi. 2010. "Experimental investigation on turbulence intensity reduction in subsonic wind tunnels." Aerospace and Technology 15: 137-147. Dol:10.1016/j.ast.2010.06.009. https://doi.org/10.1016/j.ast.2010.06.009

[6] Yong, T. H., and S. S. Dol. "Design and Development of Low-Cost Wind Tunnel for Educational Purpose." In IOP Conference Series: Materials Science and Engineering, vol. 78, no. 1, p. 012039. IOP Publishing, 2015.

https://doi.org/10.1088/1757-899X/78/1/012039 